\title{
Intermédialités
}

Histoire et théorie des arts, des lettres et des techniques

Intermediality

History and Theory of the Arts, Literature and Technologies

\section{La technique et son récit. Petite histoire de l'histoire de la photographie}

\section{André Gunthert}

Numéro 2, automne 2003

Raconter

Telling

URI : https://id.erudit.org/iderudit/1005459ar

DOI : https://doi.org/10.7202/1005459ar

Aller au sommaire du numéro

Éditeur(s)

Centre de recherche sur l'intermédialité

ISSN

1705-8546 (imprimé)

1920-3136 (numérique)

Découvrir la revue

Citer cet article

Gunthert, A. (2003). La technique et son récit. Petite histoire de l'histoire de la photographie. Intermédialités / Intermediality, (2), 101-114.

https://doi.org/10.7202/1005459ar
Résumé de l'article

Comment s'élabore le récit d'une pratique technique? En analysant l'émergence précoce d'une historiographie de la photographie, l'auteur propose de voir dans la construction du récit l'une des conditions de sa reconnaissance culturelle. Après l'examen des raisons contingentes expliquant la création immédiate d'une légitimité historique, puis du tournant institutionnel des années 1850, cet article met en lumière le rôle joué par la naissance d'un genre, l'histoire des inventions. Grâce au croisement du génie narratif d'un des principaux vulgarisateurs du XIX ${ }^{\mathrm{e}}$ siècle, Louis Figuier, avec le succès d'une entreprise éditoriale ponctuelle, la photographie se voit intégrée au corpus des connaissances utiles et dotée d'une légende qui la rapproche des grandes innovations de la période. 


\title{
La technique et son récit
}

\section{Petite histoire de l'histoire de la photographie}

\author{
ANDRÉ G UNTHERT
}

Selon Gilbert Simondon,

la culture est déséquilibrée parce qu'elle reconnaît certains objets, comme l'objet esthétique, et leur accorde droit de cité dans le monde des significations, tandis qu'elle refoule d'autres objets, et en particulier les objets techniques, dans le monde sans structure de ce qui ne possède pas de significations, mais seulement un usage, une fonction utile ${ }^{1}$.

La pertinence de cette distinction a souvent éclairé les travaux abordant l'interaction du fait culturel et du fait technique. Toutefois, ce constat à caractère anthropologique semble attribuer à la nature même des «objets » la raison de leur partage, sans interroger le processus qui a pu y conduire. En m’appuyant sur l'exemple de la photographie, qui offre un cas médian à mi-chemin de la technique et de l'esthétique, j'essaierai de montrer qu'il existe un mécanisme de l'accès au champ culturel, qui repose sur l'élaboration du récit.

Du réveille-matin à la clef électronique, du robinet aux pavés numériques ou au clavier de l'ordinateur, notre environnement quotidien est peuplé d'objets silencieux qui ressortissent au monde de l'usage évoqué par Simondon. Si chacun d'entre eux découle de processus évolutifs élaborés et parfois paradoxaux, nous avons pour l'essentiel oublié les déterminations techniques, économiques et sociales qui ont conduit aux formes et aux usages de ces divers ustensiles $^{2}$. Parmi ces objets sans histoire, certains participent pourtant d'une

1. Gilbert Simondon, Du mode d'existence des objets techniques, Paris, AubierMontaigne, 1969, p. 10.

2. Voir notamment: Gerhard Dohrn-van Rossum, L'histoire de l'heure. L'horlogerie et l'organisation moderne du temps, trad. Olivier Mannoni, Paris, Éditions de la Maison 
perception plus complexe. C'est le cas des techniques d'enregistrement iconographique - photographie, cinéma ou vidéo - qui semblent témoigner d'un statut clivé. D’un côté, nous n’interrogeons guère au-delà des paramètres du prix, du confort d'utilisation ou du design les outils de conservation du souvenir dont nous disposons à titre privé; de l'autre, nous n'ignorons pas que ces pratiques sont dotées d'un versant noble, qu'atteste leur présence dans les musées, l'édition, la recherche universitaire ou les programmes de subventions publiques.

D'où provient le statut culturel de ces technologies? La réponse semble évidente: il résulte des pratiques de création auxquelles elles ont donné lieu. Malheureusement, cette explication revient à une tautologie. Ce ne sont pas les usages artistiques qui confèrent aux techniques leur dignité culturelle, mais bien la construction préalable d'un réseau de significations qui conditionne leur emploi sur le terrain de l'art. Quoique les technologies d'enregistrement sonore proposent des fonctions strictement homologues à celles de la captation visuelle, il existe bien des festivals de photographie, de cinéma ou de vidéo, mais aucun festival du magnétophone, du microsillon ou de l'échantillonnage numérique - qui demeurent à nos yeux de purs instruments dépourvus de toute dimension symbolique.

$\mathrm{Ni}$ la nature des technologies, ni leurs usages sociaux ne suffisent à expliquer le statut actuel des pratiques d'enregistrement visuel. En revanche, il convient de noter que celui-ci n’a pas été acquis d'emblée; qu'il a notamment fallu à la plus ancienne de ces techniques plusieurs décennies avant d'accéder à une reconnaissance culturelle discernable ${ }^{3}$; que ses descendants proches ou lointains ont conquis de plus en plus vite leurs lettres de noblesse, comme si ces pratiques profitaient de leur emboîtement chronologique. Semblables observations attirent l'attention sur le caractère historique de la perception de ces objets. L'analyse du cas photographique, dans sa période primitive, permettra d'apercevoir comment s'élabore le récit d'une pratique technique - et combien celui-ci participe directement de son accession à la sphère culturelle.

des sciences de l’homme, 1997; Réseaux, numéro spécial, «Les claviers », n 87, janvierfévrier 1998; Delphine Gardey, «The Standardization of a Technical Practice: Typing (1883-1930)", History @ Technology, vol. XV, 1999, p. 313-343; Bruno Jacomy, L'âge du plip. Chroniques de l'innovation technique, Paris, Éditions du Seuil, 2002.

3. Pour la France, la reconnaissance d'utilité publique de la Société française de photographie en 1892 par le président Sadi Carnot peut constituer un repère chronologique recevable. 


\section{TEMPORALITÉS DE L'INVENTION}

Une pratique à ses commencements ne génère qu'exceptionnellement sa propre histoire. Rien ne permettait de penser que la divulgation de la technologie photographique dérogerait à cette règle. Dans son bref prospectus de 1838, Louis Daguerre ne rappelle que pour mémoire sa collaboration avec Nicéphore Niépce, se consacrant plutôt à décrire les caractéristiques et les usages du nouvel outil ${ }^{4}$. Lorsque François Arago et Jean-Baptiste Biot procèdent à la première annonce publique du daguerréotype, le 7 janvier 1839 devant l'Académie des sciences, ils se limitent eux aussi à une évocation rapide des principaux traits du procédé, sans accorder plus d'importance à l'ancien associé de Daguerre 5 . Pourquoi agiraient-ils autrement? Niépce est mort en 1833, avant la mise au point de la technologie du développement aux vapeurs de mercure - autrement dit avant l'invention de l'image latente, qui autorise la technique photographique à sortir des limbes de l'expérimentation. Daguerre, qui a longuement testé les différents aspects de son procédé, est apparemment seul à pouvoir proposer un système d'enregistrement cohérent, prêt à être mis sur le marché (fig. 1).

Ces conditions optimales se modifient avec la cascade de revendications qui suivent l'annonce, à commencer par celle de William Henry Fox Talbot, publiée dès le 9 février dans l'Athenceum ${ }^{7}$. Désireux de voir reconnaître la préexistence de ses travaux, l'érudit anglais n'en est pas moins conscient de la fragilité de sa réclamation: ses expériences de 1835 n’ont donné lieu à aucune

4. Voir Louis Daguerre, «Daguerréotype» [Paris, 1838], reproduit dans Le daguerréotype français. Un objet photographique (catalogue d'exposition), Paris, Musée d'Orsay, Réunion des musées nationaux, 2003, p. 383-384.

5. Voir François Arago, Jean-Baptiste Biot, «Physique appliquée. Fixation des images qui se forment au foyer d'une chambre obscure», Comptes rendus de l'Académie des sciences, 1839 (séance du 7 janvier), vol. 8, p. 4-7.

6. André Gunthert, «La boîte noire de Daguerre », Le daguerréotype français, p. 33-40.

7. William Henry Fox Talbot, «Some Accounts of the Art of Photogenic Drawing» [1839], reproduit dans Beaumont Newhall (éd.), Photography, Essays and Images, New York, Museum of Modern Art, 1980, p. 23-31. La contestation de Talbot sera notamment suivie par celle de Francis Bauer, publiée le 2 mars (voir Isidore Niépce, Post tenebras lux. Historique de la découverte improprement nommée daguerréotype, Paris, Astier, 1841, p. 61-67), puis par la présentation des travaux photographiques d'Hippolyte Bayard le 13 mai à Biot et le 20 mai à Arago. 
publication, ce qui lui interdit de prétendre à une antériorité en bonne et due forme. C'est donc pour rétablir l'équilibre et amoindrir la nouveauté de la proposition de son rival que Talbot fait appel au tribunal de l'histoire, suggérant que la sensibilité des sels d'argent, largement documentée dans la littérature scientifique, n'est pas une découverte. Une coïncidence heureuse va lui fournir un argument de poids. Un de ses collègues de la Royal Society, John Davy, met la dernière main à l'édition de l'œuvre complète de son père, le célèbre chimiste Humphry Davy. Cette publication qui paraît dans le courant de l'année 1839 comprend, dans son deuxième volume, la reprise d'une contribution de Thomas Wedgwood de 1802, oubliée de tous, qui mentionne un essai infructueux de fixation de l'image de la camera obscura par le nitrate d'argent ${ }^{8}$. Trop heureux de pouvoir mentionner ce précédent qui comporte toutes les garanties de scientificité, Talbot l'insère dans son article de l'Athenceum, qu'il fait aussitôt parvenir à Jean-Baptiste Biot.

Pour Arago, cette contestation ravive un scénario qu'il a expérimenté dix ans plus tôt, à l'occasion de la publication de sa Notice historique sur les machines à vapeur, fortement contestée outre-Manche ${ }^{9}$. En 1829, irrité par ces attaques, le savant avait répliqué avec «vivacité ${ }^{10} »$. Instruit par ce précédent, il corrige sa stratégie de présentation du daguerréotype. Avant de décrire les caractéristiques de la nouvelle technologie, son rapport produit le 7 juillet devant l'Assemblée nationale s'enrichit d'un long préambule historique qui, sans emprunter le ton de la polémique, répond cependant point par point aux diverses objections britanniques ${ }^{11}$. Désormais utile pour contrer la revendication de Talbot, Niépce y reparaît, ainsi que Wedgwood, cité dans le texte, que l'astronome a pris soin de vérifier à la source. Pour répliquer à son tour à l'antériorité anglaise, il exhume un exemple beaucoup moins solide, celui du

8. Thomas Wedgwood, «An Account of a Method of Copying Painting upon Glass, and of Making Profiles by the Agency of Light upon Nitrate of Silver » [1802], reproduit dans John Davy (éd.), Memoirs of the Life of Sir Humphry Davy, Londres, Smith, Elder \& Co., 1839, vol. II, p. 240-245.

9. Voir à ce sujet François Arago, «Notice historique sur les machines à vapeur » [1829], Euvres complètes, Jean Augustin Barral (éd.), Paris, Leipzig, Gide, Weigel, 1855, tome V, vol. 2, p. 1-81.

10. François Arago, «Examen des observations critiques dont la notice précédente a été l'objet» [1837], CEuvres complètes, p. 82.

11. François Arago, Rapport sur le daguerréotype, Paris, Bachelier, 1839, p. 7-19. 


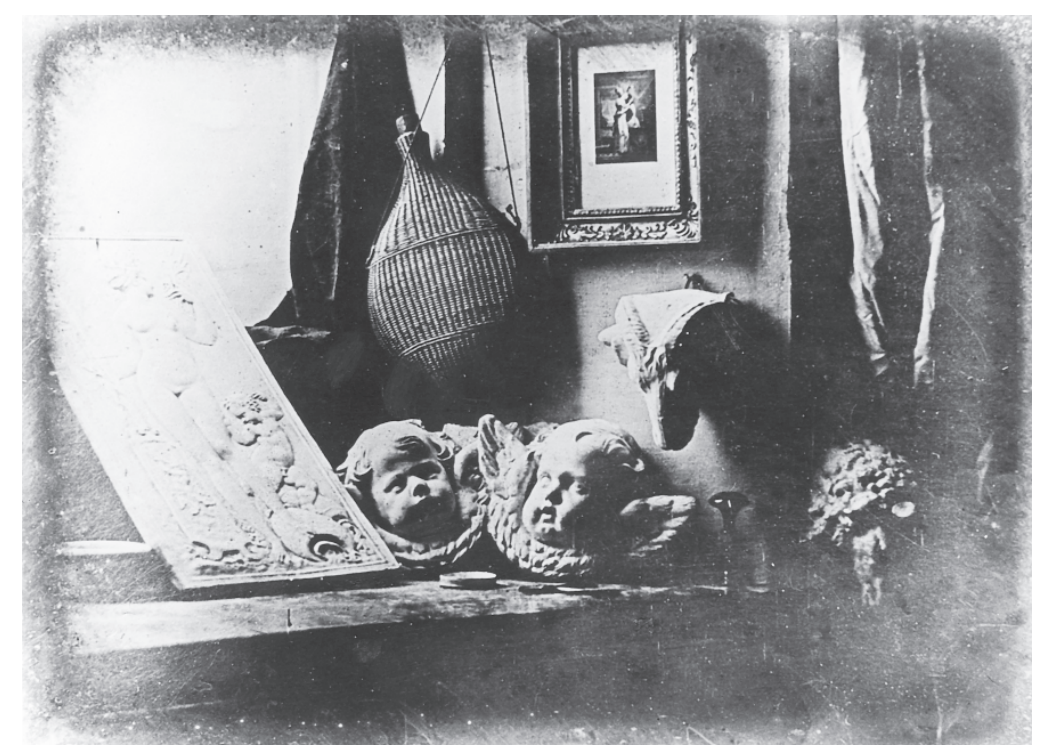

Fig. 1. Louis Daguerre, Nature morte avec bas relief d'après J. Goujon, daguerréotype, $16,5 \times 21,5 \mathrm{~cm}, 1837$ (collection Société française de photographie).

physicien Jacques-Alexandre-César Charles (1746-1822), qui aurait procédé à des essais similaires (mais non documentés ${ }^{12}$ ) dès les "premières années du XIx ${ }^{\mathrm{e}}$ siècle». Sachant que ce cas douteux ne suffira pas à l'emporter, Arago procède à un coup de force rhétorique : il invente une histoire longue dont la découverte française représenterait le point culminant. Mettant à profit sa vaste documentation d'histoire des sciences, l'astronome fait assaut d'érudition, convoque la Magia naturalis de Giambattista Della Porta $\left(1537^{-1615}\right)$ ou le De metallicis rebus de Georg Fabricius (1516-1571), classiques de l'optique et de l'alchimie, pour situer les antécédents de l'invention à l'époque de la Renaissance.

Le schéma qu'il édicte, isolant d'un côté la lignée de la camera obscura et des recherches optiques, de l'autre celle de la réaction photosensible et des travaux chimiques, fournit aujourd'hui encore le modèle de tous les chapitres introductifs des histoires générales du médium. Cette généalogie est pourtant

12. Voir Jules Lermina, «Notes pour servir à l'histoire de la photographie. III. Le physicien Charles», Le photographiste, 1904, p. 127-128 et 142-143 (je remercie Clément Chéroux de m'avoir signalé cet article). 
largement fictive, dans la mesure où les précédents évoqués n’ont pas été mobilisés par les pionniers de l'enregistrement visuel. Mais la construction produite par Arago pour des raisons essentiellement tactiques a pour effet de conférer une dignité inattendue au procédé. Plutôt qu'une curiosité de laboratoire, celui-ci apparaît comme l'aboutissement d'une longue série de recherches, inscrit dans une profondeur historique que les travaux de Daguerre seuls ne laissaient pas soupçonner. Le talent de l'un des premiers grands vulgarisateurs modernes fait de cette élaboration le point de départ d'un véritable genre: jusqu'à la fin du XIx siècle, la plupart des manuels spécialisés sacrifieront au rite de l'introduction archéologique (les moins érudits se bornant à reprendre la matière proposée par le Rapport, les plus savants s'enhardissant à corriger ou à prolonger l'exploration $\left.{ }^{13}\right)$.

\section{LES CONDITIONS DE L'HISTOIRE}

Mais la fonction inaugurale de cette préhistoire prend fin avec le lancement du daguerréotype et, passé l'affairement de la divulgation, le rappel de cette généalogie n'a plus qu'un intérêt cosmétique. Dans l’immédiat, la stratégie d’Arago aura engendré quelques effets secondaires notables, comme la refonte par Daguerre du manuel destiné à vulgariser la pratique du procédé sous l'espèce d'un dossier intitulé: Historique et description des procédés du daguerréotype et du diorama (1839), qui comporte les principales pièces justificatives susceptibles d'asseoir ses titres de paternité ${ }^{14}$. Deux ans plus tard, Isidore Niépce produira en réponse un pamphlet titré Historique de la découverte improprement nommée daguerréotype (1841), accumulant au contraire les témoignages en faveur des travaux de son père ${ }^{15}$. Mais l'heure est passée des débats sur l'invention: les activités du champ photographique sont toutes entières orientées vers la pratique. Pendant une dizaine d'années, celle-ci ne suscite aucun questionnement intellectuel autre que ceux, très spécialisés, portant sur les querelles de priorité des divers perfectionnements proposés ou sur l'explicitation des phénomènes

13. Voir respectivement, aux deux extrémités du spectre: Jean Pierre Thierry, Daguerréotypie. Franches explications... Précédées d'une histoire générale abrégée de la photographie, Paris, Lerebours et Secrétan, 1847, p. 1-45; Alexandre Ken, Dissertations historiques, artistiques et scientifiques sur la photographie, Paris, Librairie nouvelle, 1864.

14. Louis Daguerre, Historique et description des procédés du daguerréotype et du diorama, Paris, Alphonse Giroux, 1839.

15. Isidore Niépce, Post tenebras lux. 
physico-chimiques en jeu. Pourquoi en aurait-elle occasionné davantage? Placé sous le signe quasi exclusif du daguerréotype, partagé entre loisir savant, pour une poignée de riches amateurs, et activité commerciale, pour l'écrasante majorité des praticiens, l'exercice de la photographie semble, aux yeux de ses propres acteurs, ne soulever d'autres interrogations que celle de sa maîtrise procédurale. Aucun débat, aucune revendication esthétique ne marquent encore un champ uniforme qui s'est installé aux marges de la culture. Ses seules rivalités sont celles de la concurrence marchande, sa seule problématique celle du retour sur investissement. En bref, une pratique guère différente du commerce de la chaussure, dont on ne voit pas bien en quoi elle solliciterait la curiosité historique.

\section{Selon Auguste Caron:}

Lorsqu'une science, un art arrivent à l'apogée de perfection; lorsqu'il y a un ou plusieurs siècles que cet art, cette science nourrissent l'admiration universelle, c'est alors seulement qu'on songe à écrire leur histoire, qu'on s'ingénie et qu'on s'évertue à remonter aux premiers temps de leur découverte et de leur apparition [...]. Lorsque la photographie arrivera à ses derniers perfectionnements, on fera pour cet art ce qu'on a fait pour la typographie et pour tant d'autres arts; on collectionnera les produits du passé, on cherchera à préciser les dates de ces produits, à retrouver les noms des artistes dont ils [émanent]. Il est pourtant si facile de faire tout cela dès à présent ${ }^{16}$ !

Publié en 1853 sous la forme d'un appel à la création d'un «musée photographique », cet article exprime l'illusion rétrospective commune aux contemporains d'une nouvelle pratique ${ }^{17}$. Il manifeste tout autant un intérêt inédit

16. Auguste Caron, «Un musée photographique », Le propagateur, $\mathrm{n}^{\circ}$ 1, 20 novembre 1853 , n. p. [p. 2].

17. En réalité, à la date où ce texte est rédigé, nombre de documents essentiels ont déjà été dispersés ou perdus, en particulier la quasi-totalité des travaux préparatoires des deux pionniers. En l'absence de la majeure partie de la correspondance Niépce-Daguerre, déposée dans les archives de l'académie des sciences de Saint-Pétersbourg et qui ne sera éditée qu'un siècle plus tard, en 1949 (voir Toritchan Pavlovitch Kravets, éd., Documentii po istorii izobretenia fotografii, Moscou, Leningrad, Académie des sciences d'U.R.S.S., 1949; voir également Serge Plantureux, L'espion et la fougère. La mission de Joseph Hamel chez Talbot, Niépce et Daguerre, Paris, Venti, 2003), les éléments dont disposent les contemporains se réduisent peu ou prou aux deux dossiers, fortement orientés, publiés respectivement par Daguerre et Isidore Niépce (voir ci-dessus, notes 14 et 15). Le premier vise à refermer l'histoire sur l'état d'un procédé arrivé au terme de son développement; 
pour l'histoire, dont la précocité contredit son propre rappel de la théorie historiographique classique, selon laquelle la préoccupation des origines croît en proportion de leur éloignement dans le passé.

À peine plus d'une décennie après la divulgation, le début des années 1850 marque un tournant dans la perception du champ photographique. En sommeil depuis 1839, la photographie sur papier n'avait jusqu'alors mobilisé qu'un très petit nombre d'acteurs (fig. 2). La publication de procédés plus commodes et plus sûrs que celui proposé par Talbot réveille une pratique que tout oppose au daguerréotype. En 1851, un groupe de militants du négatif papier crée la Société héliographique, dont la dénomination même indique un investissement du terrain de l'histoire. Référence à l'ancien procédé de Niépce, le choix du terme «héliographie» vise non seulement à faire oublier la fondation daguerrienne de la photographie, au profit d'un patronage plus lointain et plus noble, mais aussi à prendre ses distances avec la pratique vulgaire du portrait sur plaque, incompatible avec la revendication d'une esthétique du médium ${ }^{18}$. En un mot, la nouvelle association se conçoit à la manière d'une avant-garde, rejetant dans les limbes d'un passé révolu une conception et un exercice qu'elle récuse, tout en désignant le chemin de «l'avenir ${ }^{19}{ }$, qu'elle se donne pour tâche de représenter.

Transformer la réception de la photographie, établir les conditions grâce auxquelles celle-ci pourra accéder au rang de pratique culturelle reconnue : tel est le programme de la Société héliographique. Grâce à la publication du premier périodique européen exclusivement consacré au médium, La Lumière, l'association prend le pilotage de la diffusion de l'information technique, jusquelà dispersée dans les manuels et les revues non spécialisées. Elle y adjoint l'invention d'un discours esthétique, inspiré du modèle de la critique des salons ${ }^{20}$.

le second plonge au contraire dans l'archéologie des expérimentations héliographiques pour restituer l'ampleur de l'apport du chercheur bourguignon. Entre les deux, la part manquante concerne le cœur du récit de l'invention: la collaboration fructueuse des deux associés et la mise au point du daguerréotype.

18. Pour une présentation plus détaillée, voir André Gunthert, «L'institution du photographique. Le roman de la Société héliographique », Études photographiques, ${ }^{\circ}$ 12, novembre 2002, p. 37-63.

19. Voir Gustave Le Gray, Nouveau traité théorique et pratique de photographie sur papier et sur verre, Paris, Lerebours \& Secrétan, juillet 1851, p. 9.

20. Emmanuel Hermange, «La Lumière et l'invention de la critique photographique », Études photographiques, nº 1, novembre 1996, p. 89-108. 


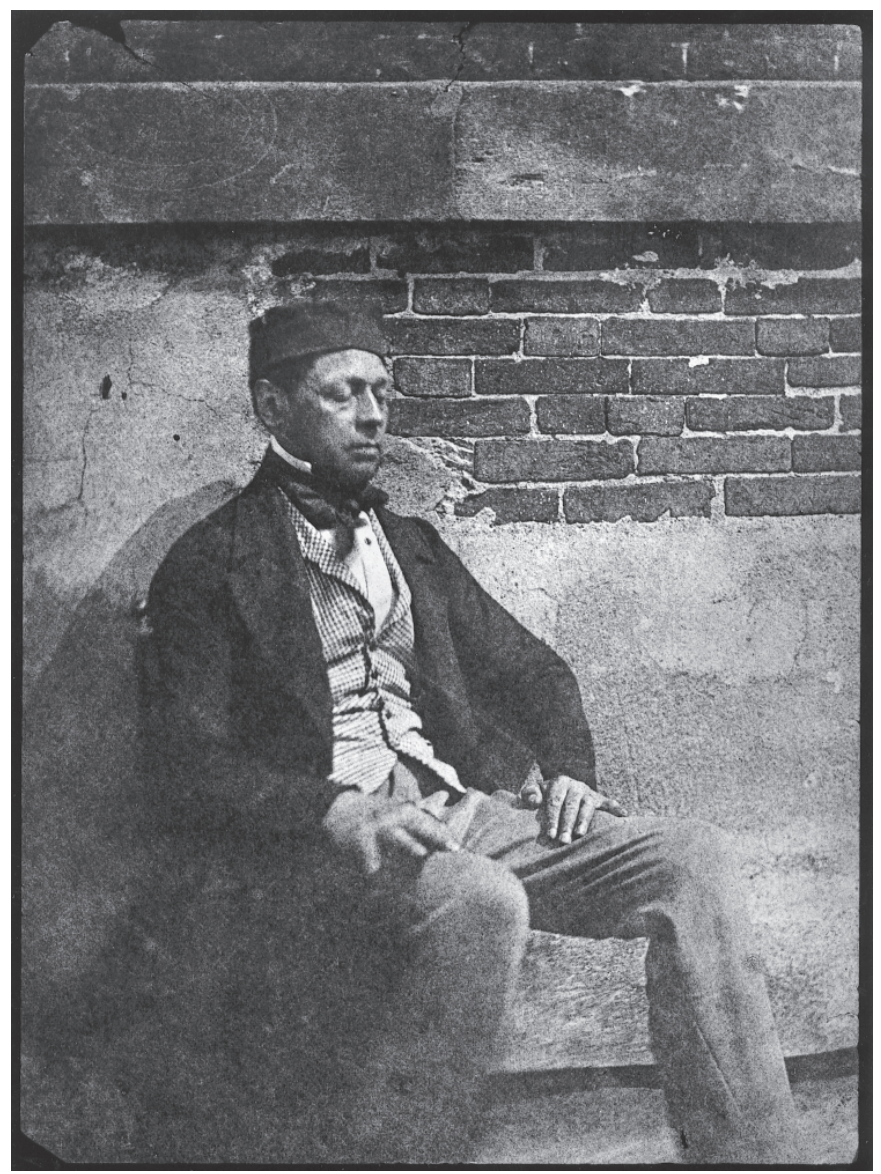

Fig. 2. Hippolyte Bayard, Autoportrait aux yeux fermés, tirage moderne d'après négatif papier, $22,4 \times 16,4 \mathrm{~cm}$, v. 1848 (collection Société française de photographie).

Elle y manifeste enfin sa préoccupation de l'établissement d'une histoire de la photographie, par l'édition de documents inédits, d'articles ou de compte rendus de livres.

C'est dans ce dernier registre qu'apparait le plus manifestement la volonté d'exercer un magistère sur l'histoire ${ }^{21}$. En août 1851, une recension de Francis Wey, l'un des principaux rédacteurs de La Lumière, accueille frâichement les

21. Voir également à ce propos: Michel Poivert, «Hippolyte Bayard et la préhistoriographie de la photographie», Revue de l'art, $\mathrm{n}^{\circ} 141$, automne 2003, p. 25-30. 
deux premiers volumes d'un ouvrage intitulé: Exposition et histoire des principales découvertes scientifiques modernes, d'un auteur encore peu connu, Louis Figuier $^{22}$. Si le critique loue l'ambition de brosser le panorama des progrès contemporains, le chapitre consacré à la photographie souffre à ses yeux de graves lacunes ${ }^{23}$. Plus encore que des omissions de détail, il reproche une information datée, qui ne tient pas compte des derniers développements du médium et empêche par conséquent de juger convenablement de son rôle esthétique.

En suggérant à l'auteur de l'Exposition... de passer d'une histoire du daguerréotype à une histoire de la photographie proprement dite, Francis Wey vient de précipiter la naissance de son historiographie. Précédée par un coup d'essai en 1848, sous la forme d'un article publié par La revue des deux mondes ${ }^{24}$, la notice sur la photographie de Figuier n'est encore en 1851 qu'une rapide synthèse des dossiers de Daguerre et d'Isidore Niépce, auxquels s'ajoutent quelques emprunts aux Comptes rendus de l'Académie des sciences ou au Traité pratique de photographie de Marc-Antoine Gaudin ${ }^{25}$. Mais dès la troisième édition de l'ouvrage, publiée en 1854 , le texte connaît une importante révision ${ }^{26}$. Passant de 71 à 122 pages, conformément aux recommandations de Wey, il s'enrichit de larges compléments consacrés à la photographie sur papier, directement puisés dans La Lumière. La chronologie de la notice n'est pas sans défauts, le paysage qu'elle brosse est loin d'être exhaustif, son style porte encore la trace de nombreux «couper-coller »; il n'empêche: plus complète et plus dense que les esquisses introductives des manuels, elle propose dès ce moment un corps de connaissances organisé, dont les grandes lignes et les principaux repères ne varieront plus - le meilleur sinon le seul récit synthétique des premiers temps de la photographie.

22. Louis Figuier, Exposition et histoire des principales découvertes scientifiques modernes, vol. I-II, Paris, Masson, Langlois \& Leclerc, $1^{\text {re }}$ édition, 1851.

23. Francis Wey, «Publications héliographiques. II », La Lumière, $1^{\mathrm{re}}$ série, $\mathrm{n}^{\circ} 26$, 3 août 1851 , p. 102-104.

24. Louis Figuier, "La photographie", La revue des deux mondes, tome XXIV, octobre-décembre 1848, p. 114-138.

25. Marc-Antoine Gaudin, Traité pratique de photographie, Paris, Dubochet, 1844.

26. Louis Figuier, "La photographie», Exposition et histoire des principales découvertes scientifiques modernes, vol. II, Paris, Masson, Langlois \& Leclerc, $3{ }^{\mathrm{e}}$ édition, 1854 , p. 1-122. 


\section{DE L'IMAGE À L'IMAGINAIRE}

Parmi les vulgarisateurs de la seconde moitié du XIX ${ }^{\mathrm{e}}$ siècle ${ }^{27}$, quelques-uns, tels François Moigno ou Gaston Tissandier, s'illustrent par une pratique assidue de la photographie, ce qui explique en partie leur intérêt pour la spécialité. Tel n'est pas le cas de Louis Figuier, dont la confrontation avec le médium est pourtant l'une des plus précoces. Né en 1819 à Montpellier où il obtient son doctorat de médecine et son agrégation de pharmacie, le chercheur poursuit ses études à Paris sous la direction du célèbre chimiste Antoine Jérôme Balard, futur président de la Société française de photographie, et consacrera en 1850 sa thèse de doctorat ès sciences à l'Action de la lumière sur quelques substances impressionnables. Quoique ce bagage constitue à l'évidence un viatique précieux pour s'aventurer en territoire photographique, la contribution de Figuier à l'histoire du médium n'en reste pas moins la première proposée par un non-spécialiste.

Les composantes de l'innovation scientifique et technique sont rarement accessibles d'emblée au grand public. Premier auteur à s'être délibérément confronté à l'option de la vulgarisation, François Arago, tout au long de la rédaction de ses Notices biographiques et scientifiques, avait testé plusieurs moyens susceptibles de faciliter la communication de l'information technique, en particulier l'anecdote, la métaphore et la mise en perspective historique. En s'inspirant de ce modèle, Figuier le systématise : alors que les essais du savant se déployaient au gré de ses travaux, les articles du compilateur forment un corpus encyclopédique supposé rassembler l'essentiel des progrès scientifiques et techniques de son époque. Ce projet fonde simultanément un genre et sa méthode : l'histoire des inventions - ou comment familiariser par l'intermédiaire du récit historique un public non spécialisé avec l'innovation scientifique et technique ${ }^{28}$.

L'inclusion de la photographie, dès 1851, au sein de ce catalogue où elle voisine avec le chemin de fer, la pile de Volta, la télégraphie, l'éclairage au gaz et les aérostats, constitue l'un des principaux facteurs qui la préserve d'une perception strictement utilitaire. Encore fallait-il que le propos de Figuier puisse être partagé par un large public. Plus que le bon accueil réservé à l'Exposi-

27. Voir Bruno Béguet (dir.), La science pour tous. Sur la vulgarisation scientifique, 1850-1914, Paris, Bibliothèque du Centre national des Arts et métiers, 1990; Daniel Raichvarg, Jean Jacques, Savants et ignorants. Une histoire de la vulgarisation des sciences, Paris, Éditions du Seuil, 1991.

28. Michel Constantial, Gilles Eboll, Jean-François Foucaud et al., Le savant du foyer. Louis Figuier et la vulgarisation scientifique au XIX $X^{e}$ siècle (catalogue d'exposition), Paris, Fédération française de coopération entre bibliothèques, 1993. 
tion..., qui connaîtra cinq réimpressions, l'intégration de ce corpus à l'un des plus gros succès de librairie du dernier tiers du XIX ${ }^{\mathrm{e}}$ siècle va en démultiplier considérablement l'impact. Depuis les années 1830, l'édition française a apprivoisé la publication illustrée de gravures ${ }^{29}$. L'investissement important que suppose ce type d'entreprise la réserve aux projets populaires, comme les romans de Walter Scott, illustrés par Tony Johannot, ou les ouvrages historiques d'Adolphe Thiers, d'Alphonse de Lamartine ou d'Augustin Thierry. En publiant en 1867 au format in-quarto le premier volume des Merveilles de la science, les éditeurs Furne et Jouvet inaugurent le mariage de la vulgarisation scientifique avec l'image - formule dont la prospérité continue aujourd'hui d'assurer les beaux jours de quelques maisons spécialisées ${ }^{30}$.

L'illustration gravée parachève le système narratif mis en place par Figuier. Régulièrement ponctué des démonstrations d'érudition nécessaires à la crédibilité du genre, son récit reste basé sur l'anecdote et la dramatisation, scandé par le dialogue en style direct, dans le but de provoquer la confrontation la plus immédiate avec l'événement rapporté31. Pour le dessinateur formé à l'illustration de pièces de théâtre, de romans ou d'ouvrages historiques, le texte du vulgarisateur offre une trame familière : à côté de la reproduction des dispositifs ou des portraits des acteurs, l'iconographie des Merveilles de la science fait la part belle aux scènes reconstituées (fig. 3), à la «figuration » d'incidents, voire à l'évocation fictive.

29. Voir notamment Thierry Gervais, «D'après photographie. Premiers usages de la photographie dans le journal L'Illustration (1843-1859) ", Études photographiques, n ${ }^{\circ}{ }_{13}$, juillet 2003 , p. 58-60.

30. Voir Louis Figuier, Les merveilles de la science, Paris, Furne, Jouvet et Cie, 6 vol., 1867-1891.

31. Un seul exemple: dans un article de 1854, Francis Wey évoque, d'après une anecdote qui lui a été personnellement rapportée par Charles Chevalier, un «inventeur inconnu»: «Le [jeune homme] sourit, et tirant de sa poche une enveloppe pliée, il en sortit une image sur papier, en disant: "Voilà ce que j'ai obtenu en plaçant mon objectif devant la fenêtre de ma chambre." » (François Wey, «Comment le soleil est devenu peintre. Histoire du daguerréotype et de la photographie. 1", Musée des familles, vol. XX, juin 1853, p. 263; voir également: Charles Chevalier, «Troisième partie. Documents historiques ", Guide du photographe, Paris, chez l'auteur, 1854, p. 21) Le même passage devient chez Figuier: "Pour toute réponse, le jeune homme tira de sa poche un vieux portefeuille usé et rapiécé. Dans ce portefeuille, il prit une feuille de papier enveloppée avec soin; puis, la dépliant, il la plaça sur la vitrine de l'opticien: "Voilà ce que je puis obtenir", dit-il avec simplicité. » (Louis Figuier, «La photographie», dans Les merveilles de la science, vol. III, 1868, p. 20) 


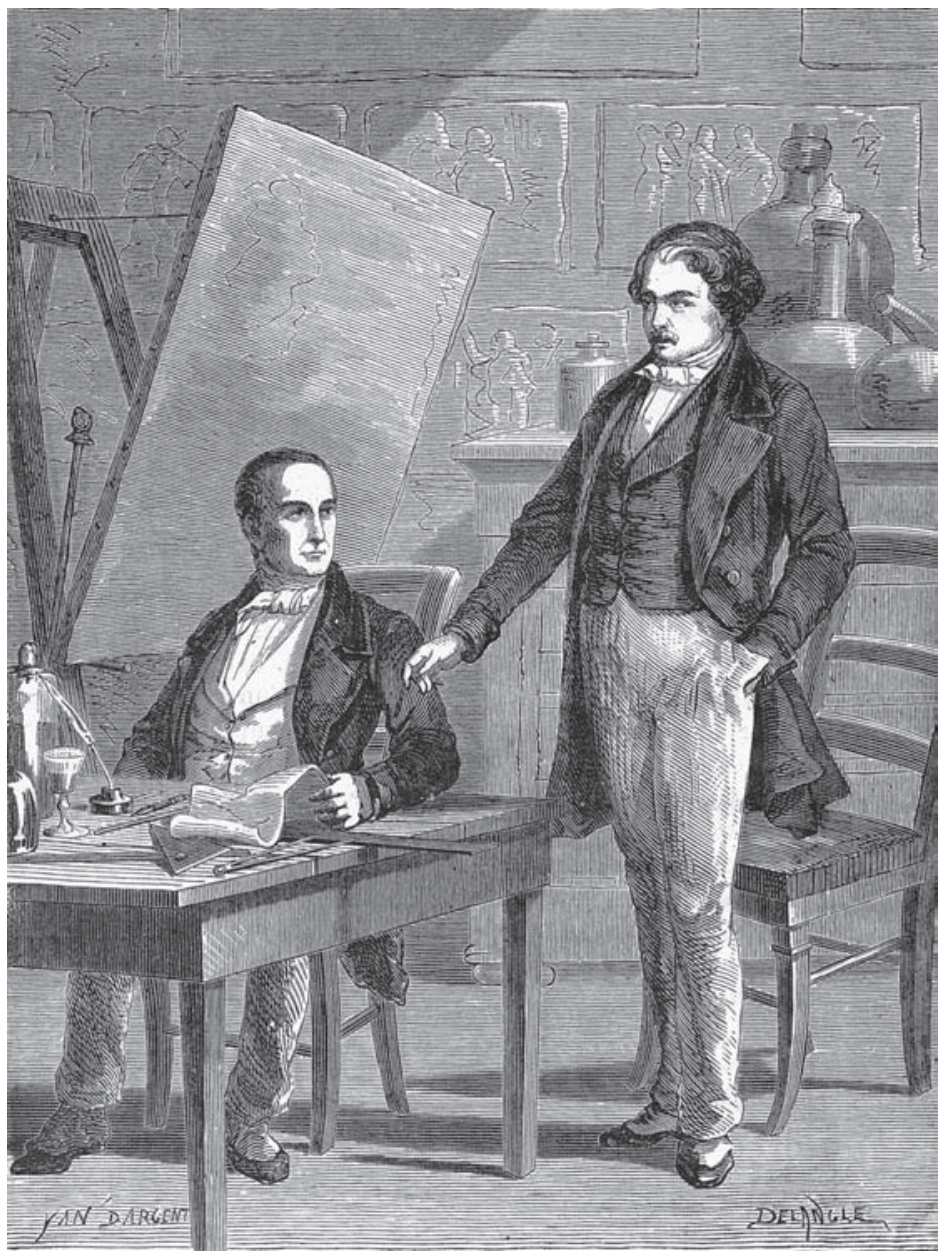

Fig. 3. Yan d'Argent, "Niépce lisant à Daguerre, après leur association, la description de son procédé ", illustration pour Les merveilles de la science, tome III, 1868, p. 33 (coll. part.).

Présent dans toutes les bibliothèques des familles bourgeoises de la III République, où il voisine avec le Voyage autour du monde de Dumont d'Urville ou les romans illustrés de Jules Verne, l'ouvrage de Figuier offre au public cultivé la fresque qui intègre la technique et la science au corpus nécessaire des savoirs citoyens. Destiné par son iconographie à des fins pédagogiques, il peuple l'imaginaire des jeunes générations par ses descriptions saisissantes et ses illustrations théâtrales. Un réflexe propre aux succès de librairie parachève 
la stabilité de la proposition: si le succès de la formule encourage la récidive, il favorise aussi la répétition d'un contenu qui a fait ses preuves. Cette forme de paresse éditoriale explique que la reproduction sonore, exclue par Figuier, et dont les premières histoires développées paraissent après $1900^{32}$, demeure absente des grandes compilations de la première moitié du $\mathrm{Xx}^{\mathrm{e}}$ siècle ${ }^{33}$.

Ce n'est pas par l'érudition savante que la photographie accède à la culture. L'exemple d'Arago avait établi la légitimité de l'interrogation historique à l'endroit du médium. Encouragé par les débats de La Lumière, Figuier l'extrait du champ de la spécialité et lui accorde une place de choix dans son panorama des inventions. Comme le montre la comparaison avec d'autres entreprises contemporaines, la présence de la photographie dans cet ensemble n'allait pas de soi. La précocité et la popularité de cet accueil furent autant d'atouts favorisant un processus de reconnaissance culturelle. Mais ne nous y trompons pas: la fortune de la photographie ne fut pas seulement de figurer entre les aérostats et les poudres de guerre dans un ouvrage grand public. C'est parce que le médium a pu susciter une légende haute en couleurs, digne des inventions majeures de la période, qu'il a mérité d'entrer dans la série. En d'autres termes, c'est l'économie même de la narration qui a produit les ajustements nécessaires à cette métamorphose. Figuier brosse le tableau d'une histoire humaine, accessible, débarrassée de ses subtilités techniques, émaillée de hauts faits et d'incidents apparemment mineurs, auxquels le récit confère une valeur métaphorique: c'est cette histoire et son prolongement iconographique qui font de la photographie un modèle des "principales découvertes scientifiques» du XIX ${ }^{\mathrm{e}}$ siècle.

32. Voir notamment Émile Gautier, Le phonographe, son passé, son présent, son avenir, Paris, Éditions Flammarion, 1905.

33. Voir notamment la collection d'ouvrages de luxe publiés entre 1932 et 1945 par L'Illustration, globalement fidèle à la structure proposée par Figuier, qui s'ouvre sur une Histoire de l'aéronautique et se referme avec le volume consacré à la photographie. Voir Raymond Lécuyer, Histoire de la photographie, Paris, Baschet, 1945. 